\title{
Preparation and Characterization of Poly(2-vinylpyridine)s with Narrow Molecular Weight Distributions
}

\author{
Yushu Matsushita, Kunio ShIMIZU, Yasushi NAKaO, \\ Haruhisa CHOSHI, Ichiro NODA, \\ and Mitsuru NAGASAWA \\ Department of Synthetic Chemistry, Faculty of Engineering, Nagoya University, \\ Furo-cho, Chikusa-ku, Nagoya 464, Japan
}

(Received November 14, 1985)

\begin{abstract}
Anionic polymerization of 2-vinylpyridine was carried out in tetrahydrofuran at $-78^{\circ} \mathrm{C}$ with four kinds of initiators, i.e., $n$-butyllithium, tritylsodium, cumylpotassium and cumylcesium. It was confirmed that the polymerizations with all these initiators proceeded on a living mechanism and the polymers obtained had narrow molecular weight distributions. The triad isotactic contents of the polymers prepared were almost identical, independently of counter ions. The intrinsic viscosity-molecular weight relationships were determined in two good solvents and in a $\theta$-solvent. From those relationships, it was concluded that poly(2-vinylpyridine) has almost the same chain flexibility as ordinary vinyl polymers.

KEY WORDS Poly(2-vinylpyridine) / Anionic Polymerization / Narrow Molecular Weight Distribution / Intrinsic Viscosity /
\end{abstract}

It is needless to stress the importance of the samples having sharp molecular weight distributions in studying the physical properties of polymers on the molecular basis. In particular, polar polymer samples covering a wide range of molecular weight and having narrow molecular weight distributions are highly desired for various purposes. In general, however, the living anionic polymerization of polar monomers is not so easy because of occurrence of undesired side reactions. For example, the attack of butyllithium on carbon$\mathrm{yl}$ double bond in the anionic polymerization of methyl methacrylate ${ }^{1}$ and the metallation of acrylonitrile monomer with alkali metal ${ }^{2}$ were pointed out. In the case of 4-vinylpyridine, poly(4-vinylpyridine) is not soluble in most solvents used for anionic polymerization if the molecular weight is high, so that the polymerization solution may become heterogeneous during propagation. ${ }^{3,4}$

Poly(2-vinylpyridine) (P-2VP) attracted our interest since it is a typical polar polymer and also its anionic living polymerization appears to be feasible. ${ }^{3}$ In practice, Fontanille and Sigwalt $^{5}$ obtained P-2VP samples with fairly narrow molecular weight distributions. However, the molecular weights of P-2VP obtained were rather limited $\left(\max 3 \times 10^{5}\right)$. In this paper, we would like to report anionic polymerization results of $\mathrm{P}-2 \mathrm{VP}$ having narrow molecular weight distributions and covering a wide range of molecular weight as well as their viscosity-molecular weight relationships in good and poor solvents.

\section{EXPERIMENTAL}

\section{Reagents and Polymerization}

Tetrahydrofuran (THF), used as solvent for polymerization, was purified by the same method as in previous works. ${ }^{6 a}$ All initiators, $n$-butyllithium, tritylsodium, cumylpotassium and cumylcesium, were synthesized by the 


\section{Y. Matsushita et al.}

methods reported in the literature. ${ }^{6}$ 2Vinylpyridine monomer used is of the special grade of Tokyo-Kasei Co., Ltd. It was dried over calcium hydride, distilled, treated with benzophenone-sodium and finally purified with dipotassium salt of $\alpha$-methylstyrene tetramer under reduced pressure. The last procedure was carried out just before polymerization and the monomer thus purified was immediately diluted with solvent.

Polymerization of 2-VP was carried out in vacuo at $-78^{\circ} \mathrm{C}$ in all cases. The active ends were terminated with methanol after completion of the polymerization reaction. The polymers obtained were purified by precipitation from polymerization solutions with $n$ hexane. No further purification or fractionation of the samples obtained was carried out.

\section{Characterization}

Number-averaged molecular weights $M_{n}$ of the samples were determined by osmometry using a Hewlett-Packard . High Speed Membrane Osmometer Type-502 in THF at $25^{\circ} \mathrm{C}$. Weight-averaged molecular weights $M_{w}$ and second virial coefficients were determined by light scattering with an Automatic Light Scattering Photometer Fica-50 in benzene. The wave length used was $436 \mathrm{~nm}$. Optical purification was carried out with $0.4 \mu \mathrm{m} \mathrm{Nu}$ clepore filter. The refractive index increment of P-2VP in benzene, $\partial n / \partial c$, was already determined by Arichi et al. ${ }^{7}$

GPC chromatograms of the samples were obtained by a Toyo-Soda High Speed Liquid Chromatograph HLC-802A with high resolution columns GMHXL $\times 2$. Tetramethylethylenediamine was added to the eluent (THF) by $5 \%$ to avoid the adsorption of $\mathrm{P}$ 2VP on polystyrene gels. The calibration of the columns used was carried out with Pressure Chemical Standard Polystyrenes. The eluent THF is a common good solvent for P2VP and polystyrenes.

Triad isotactic contents of the samples were calculated from their ${ }^{1} \mathrm{H}-\mathrm{NMR}$ spectra ob- tained in deuterated chloroform solutions with a JEOL FX-90Q.

\section{Intrinsic Viscosity Measurements}

Viscometers of a modified Ubbelohde type were used to measure the intrinsic viscosities of P-2VP in 2-propanol, pyridine and benzene. Both 2-propanol and pyridine are good solvents for P-2VP, while benzene is a poor solvent.

2-Propanol and pyridine, both of the special grades of Kishida-Kagaku, were fractionally distilled with and without magnesium ribbon, respectively, just before use. THF, of the special grade of Kishida-Kagaku, was dried over sodium wire, while benzene, of the spectroscopic grade of Kanto-Kagaku Cica-Merck, was used without further purification.

\section{RESULTS AND DISCUSSION}

\section{Polymerization}

Polymerization conditions and results are summarized in Tables I and II, respectively. Conversions are $100 \%$ within experimental errors in all cases. It is observed in Table II that initiation efficiencies (IE), that is, the ratios of observed molecular weights to the ones calculated from the amounts of monomer and initiator used, are sufficiently close to unity independently of counter ion species, if we take into consideration the experimental errors in determining their amounts. Some examples of GPC chromatograms of the samples obtained are shown in Figure 1. The polydispersity indices $M_{w} / M_{n}$ calculated from the GPC chromatograms, using a master curve calibrated with Pressure Chemical Standard Polystyrenes, are shown in Table II. Since the eluent (THF) is a common good solvent for the samples and the standard polymers, the ratios $M_{w} / M_{n}$ thus calculated are expected to be close to the true values, though respective values of $M_{n}$ and $M_{\mathrm{w}}$ calculated from GPC chromatographs may contain some errors. The values of $M_{w} / M_{n}$ in Table II show that the 


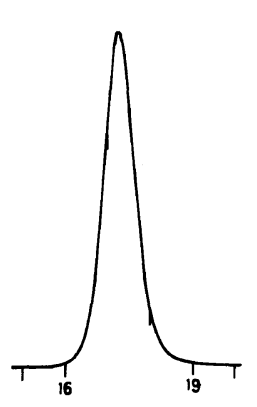

a
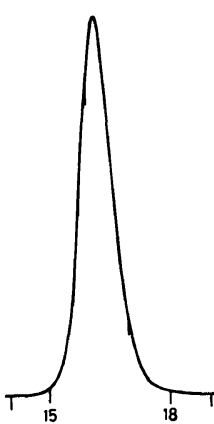

b

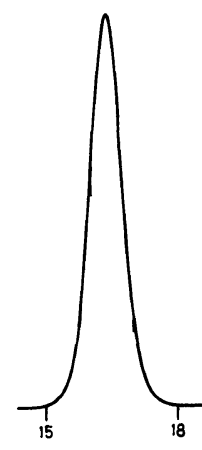

C

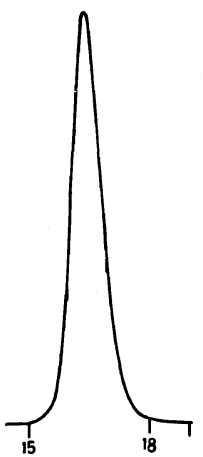

d

Figure 1. GPC chromatograms of the samples prepared with four different initiators. a) $n$-butyllithium, b) tritylsodium, c) cumylpotassium, and d) cumylcesium.

Table I. Polymerization conditions

\begin{tabular}{|c|c|c|c|c|}
\hline \multirow{2}{*}{$\begin{array}{c}\text { Sample } \\
\text { code }\end{array}$} & \multirow{2}{*}{ Initiator } & $\begin{array}{l}\text { Reaction } \\
\text { time }\end{array}$ & $\begin{array}{l}\text { Concn of } \\
\text { monomer }\end{array}$ & $\begin{array}{l}\text { Concn of } \\
\text { initiator }\end{array}$ \\
\hline & & $\mathrm{h}$ & $\mathrm{moll}^{-1}$ & $\times 10^{4} \mathrm{moll}^{-1}$ \\
\hline VPL-1 & n-BuLi & 4 & 0.37 & 4.4 \\
\hline VPN-1 & $\phi_{3} \mathrm{CNa}$ & 3 & 0.37 & 2.8 \\
\hline VPK-2 & Cumyl-K & 7 & 0.29 & 0.98 \\
\hline VPC-1 & Cumyl-Cs & 3 & 0.24 & 2.5 \\
\hline
\end{tabular}

molecular weight distributions of the samples are narrow. Summarizing all these results, we may conclude that the polymerization reaction of 2-VP in THF proceeds on a living mechanism.

In anionic polymerization, in general, an active chain end forms various types of complex with counter-ions depending on the counter-ion species, so that the tacticities of the polymers obtained are, in general, different with different counter-ion species. The ionpairs of poly(2-vinylpyridinyl) anion with different counter-ions are also in different states at room temperature. That is, sodium ion in its ion-pair with poly(2-vinylpyridinyl) anion is intramolecularly solvated with pyridine group at room temperature, ${ }^{8}$ whereas such intramolecular solvations are not observed in ion-pairs of larger ions such as $\mathrm{K}^{+}$and $\mathrm{C}_{\mathrm{s}}^{+} \cdot{ }^{9} \cdot$ However,
Table II. Polymerization results

\begin{tabular}{cclcl}
\hline $\begin{array}{c}\text { Sample } \\
\text { code }\end{array}$ & $\begin{array}{c}M_{n}{ }^{\mathrm{a}} \\
\times 10^{-4}\end{array}$ & $\mathrm{IE}^{\mathrm{b}}$ & $M_{w} / M_{n}$ & $\frac{I^{\mathrm{c}}}{\%}$ \\
\hline VPL-1 & $8.75(8.45)$ & 0.97 & 1.03 & 40 \\
VPN-1 & $17.1(14.0)$ & 0.82 & 1.10 & 46 \\
VPK-2 & $20.4(21.9)$ & 1.0 & 1.00 & 50 \\
VPC-1 & $13.1(9.7)$ & 0.74 & 1.07 & 51 \\
\hline
\end{tabular}

a Parentheses show the values calculated from the amount of monomer and initiator used.

b Initiation efficiencies.

c Triad isotactic content.

the intramolecular solvation of sodium ion disappears if the temperature is decreased to $-70^{\circ} \mathrm{C}^{8}$ The last column in Table II shows the triad isotactic contents of the samples polymerized at $-78^{\circ} \mathrm{C}$, which were estimated from ${ }^{1} \mathrm{H}$ NMR spectrum using the assignments of Matsuzaki et al. ${ }^{10}$ No significant differences in tacticity are found among the samples prepared with different initiators, as expected.

\section{Intrinsic Viscosity-Molecular Weight Relation- ship}

In addition to the samples in Table II, several P-2VP samples having higher molecular weights were prepared to determine the intrinsic viscosity-molecular weight relationship for P-2VP. Their number- and weight- 
Table III. Molecular characteristics and intrinsic viscosities of samples

\begin{tabular}{|c|c|c|c|c|c|c|}
\hline \multirow{2}{*}{$\begin{array}{l}\text { Sample } \\
\text { code }\end{array}$} & \multirow{2}{*}{$\begin{array}{c}M_{n} \\
\times 10^{-4}\end{array}$} & \multirow{2}{*}{$\begin{array}{c}M_{w} \\
\times 10^{-4}\end{array}$} & \multirow[b]{2}{*}{$M_{w} / M_{n}$} & \multicolumn{2}{|c|}{$[\eta]$} & \multirow{2}{*}{$\begin{array}{c}{[\eta]_{\theta}} \\
\text { Benzene } \\
11.4^{\circ} \mathrm{C}\end{array}$} \\
\hline & & & & $\begin{array}{l}\text { 2-Propanol } \\
25^{\circ} \mathrm{C}\end{array}$ & $\begin{array}{l}\text { Pyridine } \\
25^{\circ} \mathrm{C}\end{array}$ & \\
\hline VPL-1 & 8.75 & $9.4^{\mathrm{a}}$ & 1.08 & 0.378 & 0.405 & 0.261 \\
\hline VPK-2 & 20.4 & $20.4^{\mathrm{a}}$ & 1.00 & 0.652 & - & 0.367 \\
\hline VPL-6 & 29.6 & $30.5^{\mathrm{a}}$ & 1.03 & 0.811 & 0.970 & 0.431 \\
\hline VPL-5 & 60 & 67 & 1.14 & 1.44 & 1.68 & 0.667 \\
\hline VPK-3 & - & 196 & 1.07 & 3.15 & 3.71 & 1.18 \\
\hline
\end{tabular}

a The values were determined by multiplying $M_{n}$ by $M_{w} / M_{n}$.

averaged molecular weights and also the polydispersity index $M_{w} / M_{n}$ determined by the same methods as in Table II, are listed in Table III. $M_{w}$ with asterisks in Table III were determined by multiplying the experimental values of $M_{n}$ by $M_{w} / M_{n}$.

If we construct a master curve for P-2VP by plotting the peak elution of P-2VP against $M_{w}$ in Table III, the resulting master curve is parallel to but slightly above the master curve determined with standard polystyrenes. Use of this master curve for P-2VP gives little effect on the calculated values of $M_{w} / M_{n}$. That is, it may be concluded that the values of $M_{w} / M_{n}$ are insensitive to the choice of standard polymers if the eluent is a common good solvent for both polystyrene and P-2VP.

Intrinsic viscosities of the samples were obtained by using two different extrapolation. methods of Huggins ${ }^{11}$ and Kraemer. ${ }^{12}$ The values determined in good and poor solvents are listed in Table III. Double logarithmic plots of $[\eta]$ and $M_{w}$, for P-2VP in pyridine and 2-propanol are shown in Figure 2. The MarkHouwink-Sakurada equations thus determined are

$$
\begin{aligned}
& {[\eta]=0.99 \times 10^{-4} M_{w}^{0.73} \quad \text { (in pyridine) }} \\
& {[\eta]=1.84 \times 10^{-4} M_{w^{\prime}}^{0.67} \quad \text { (in 2-propanol) }}
\end{aligned}
$$

Thus, pyridine is a typical good solvent, while 2-propanol is a somewhat poorer solvent for P-2VP.

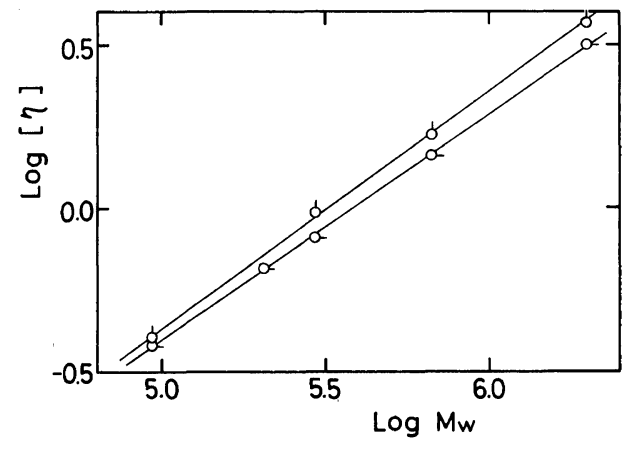

Figure 2. Double logarithmic plots of $[\eta]$ and $M_{w}$ in good solvents. $\left(\mathrm{O}^{\prime}\right)$ in pyridine, $\left(\mathrm{O}^{-}\right)$in 2-propanol at $25^{\circ} \mathrm{C}$.

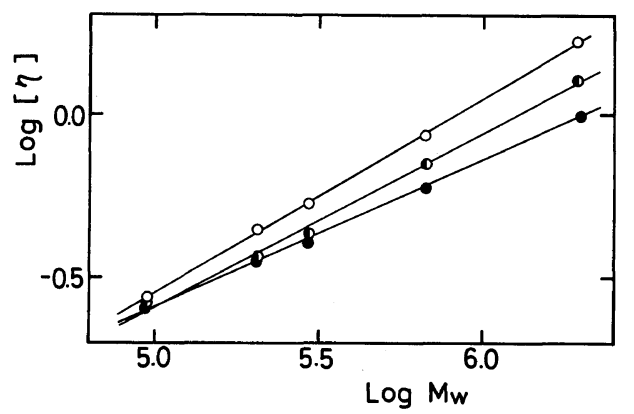

Figure 3. Double logarithmic plots of $[\eta]$ and $M_{w}$ in a poor solvent (benzene) at different temperatures. The constants in $[\eta]=K \cdot M_{w}^{\alpha}$ : (O) $15^{\circ} \mathrm{C}, K=3.18 \times 10^{-4}$, $\alpha=0.59$; (O) $12^{\circ} \mathrm{C}, K=5.60 \times 10^{-4}, \alpha=0.53$; (O) $10^{\circ} \mathrm{C}$, $K=1.49 \times 10^{-3}, \alpha=0.43$.

$[\eta]$ in benzene at three different temperatures are plotted against $M_{w}$ in Figure 3. The slope in the plots decreases with decreasing temperature and becomes 0.5 at a temperature between 


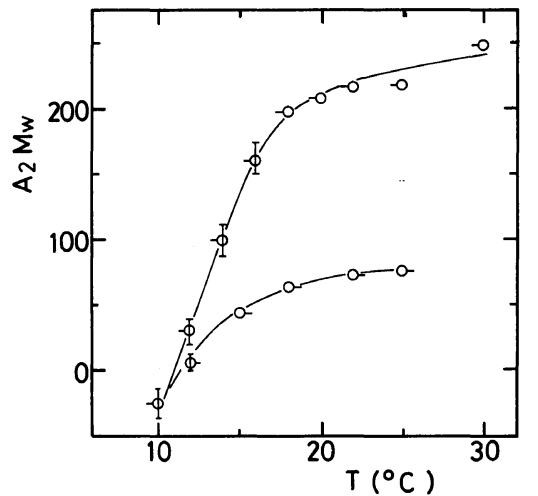

Figure 4. Second virial coefficient $v s$. temperature plots for two samples in benzene. (-O) VPL-5 and (O-) VPK3.

$10^{\circ} \mathrm{C}$ and $12^{\circ} \mathrm{C}$. The second virial coefficients of two samples determined by LS are plotted against temperature in Figure 4. From this figure, it can be confirmed that the $\theta$-temperature in benzene is $c a .11^{\circ} \mathrm{C}$. If we determine $[\eta]$ of five samples at $11.4^{\circ} \mathrm{C}$ in benzene by interpolation of experimental data at three different temperatures in Figure 3, as listed in Table III, we have

$[\eta]=8.1 \times 10^{-4} M_{w^{\prime}}^{0.50} \quad\left(\right.$ at $11.4^{\circ} \mathrm{C}$ in benzene $)$

This $\theta$-temperature considerably differs from those obtained by Dondos ${ }^{13}\left(15^{\circ} \mathrm{C}\right)$ and Arichi ${ }^{14}\left(16.2^{\circ} \mathrm{C}\right)$. The difference may arise from differences either in purity of the solvents used or in tacticity of the samples obtained.

Figure 5 shows the following StockmayerFixman plot $^{15}$ for the viscosity data in benzene at different temperatures.

$$
[\eta] / M_{w}^{1 / 2}=K_{0}+\mathrm{C} \phi_{0} B M_{w}^{1 / 2}
$$

where $K_{0}$ is related to the unperturbed mean square end-to-end distance $\left\langle r^{2}\right\rangle_{0}$ by $K_{0}=$ $\phi_{0}\left(\left\langle r^{2}\right\rangle_{0} / M\right)^{3 / 2}, \mathrm{~B}$ and $\mathrm{C}$ being constant. In Figure 5 , we again have $K_{0}=8.1 \times 10^{-4}$ $\left(\mathrm{dl} \mathrm{g}^{-1}\right)$. The data in good solvents also tend to a similar value as shown in Figure 5.

If we assume that the Flory coefficient $\phi_{0}$ is $2.5 \times 10^{21}$ and the characteristic ratio for freely jointed vinyl polymer chains $\left\langle\dot{r}^{2}\right\rangle_{\text {of }} / M$ is

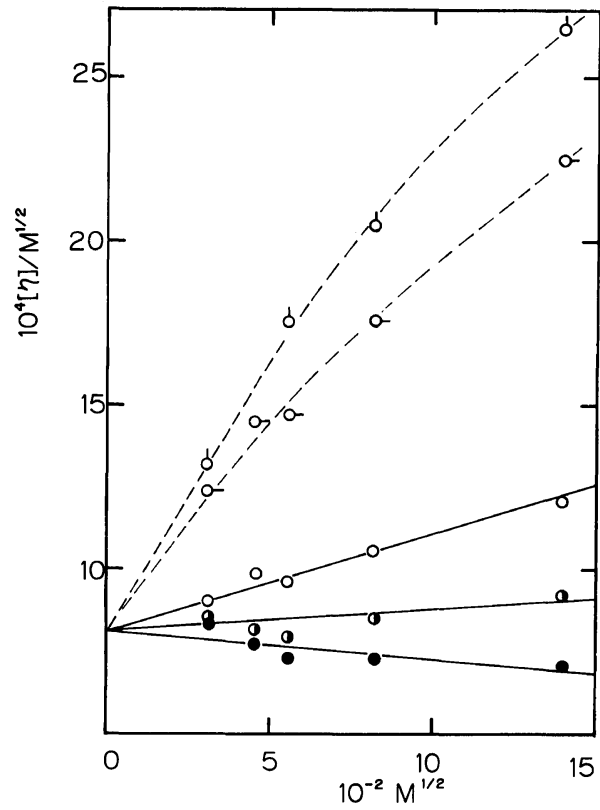

Figure 5. Stockmayer and Fixman's plots for intrinsic viscosity data in benzene. Symbols are the same as in Figures 2 and 3.

$9.06 \times 10^{-4} \mathrm{~nm}^{2}$, the so-called short range interaction parameter $\sigma\left(\equiv\left[\left\langle r^{2}\right\rangle_{0} /\left\langle r^{2}\right\rangle_{\text {of }}\right]^{1 / 2}\right)$ becomes 2.3 , which is a typical value for vinyl polymers. Dondos reported appreciably smaller values for $K_{0}$ and $\sigma$, while Arichi et al. reported almost the same value for $\sigma$ as in this work, by giving the polydispersity correction to $K_{0}$.

\section{REFERENCES}

1. N. Kawabata and T. Tsuruta, Macromol. Chem., 86, 231 (1965); K. Hatada, T. Kitayama, S. Okahata and H. Yuki, Polym. J., 13, 1045 (1981).

2. N. Kawabata and T. Tsuruta, Macromol. Chem., 98, 262 (1966).

3. C. L. Lee, J. Smid, and M. Szwarc, Trans. Faraday Soc., 59, 1192 (1963).

4. P. P. Spiegelman and G. Parravano, J. Polym. Sci., $A, 2,2245$ (1964).

5. M. Fontanille and P. Sigwalt, Bull. Soc. Chim. Fr., 4083 (1967).

6. a) T. Fujimoto, N. Ozaki, and M. Nagasawa, $J$. Polym. Sci., A, 3, 2259 (1965). b) T. Fujimoto and M. Nagasawa, Polym. J., 7, 397 (1975). c) B. Ziegler and K. Schnell, Ann., 437, 255*(1953). d) K. Ziegler and 


\section{Y. Matsushita et al.}

H. Dislich, Ber., 90, 1107 (1957).

7. S. Arichi, M. Yoshida, and Y. Ogawa, Polym. Prepr. Jpn., 23, 486 (1974).

8. M. Fischer and M. Szwarc, Macromolecules, 3, 23 (1970).

9. M. Tardi and P. Sigwalt, Eur. Polym. J., 8, 151 (1972).

10. K. Matsuzaki, T. Kanai, T. Matsubara, and S.
Matsumoto, J. Polym. Sci., Polym. Chem. Ed., 14, 1475 (1976).

11. M. L. Huggins, J. Am. Chem. Soc., 64, 2716 (1942).

12. E. O. Kraemer, Ind. End. Chem., 30, 1200 (1938).

13. A. Dondos, Macromol. Chem., 135, 181 (1970).

14. S. Arichi, Bull. Chem. Soc. Jpn., 39, 439 (1966).

15. W. H. Stochmayer and M. Fixman, J. Polym. Sci., $C, \mathbf{1}, 137$ (1963). 